\title{
Perda auditiva induzida por ruído e hipertensão em condutores de ônibus
}

\section{Noise-induced hearing loss and high blood pressure among city bus drivers}

\author{
Heleno Rodrigues Corrêa Filho ${ }^{a}$, Luciana Scarlazzari Costa ${ }^{a}$, Eduardo Luiz Hoehnea , Marco \\ Antonio Gomes Pérez ${ }^{b}$, Lilian Cristine Ribeiro Nascimento ${ }^{b}$ e Erly Catarina de Moura \\ Departamento de Medicina Preventiva e Social da Universidade Estadual de Campinas (Unicamp), \\ Campinas, SP, Brasil. ${ }^{b}$ Centro de Referência de Saúde do Trabalhador, Campinas, SP, Brasil. \\ 'Departamento de Nutrição da Faculdade de Ciências Médicas da Pontifícia Universidade Católica \\ de Campinas, Campinas, SP, Brasil
}

\section{Descritores}

Perda auditiva provocada por ruído, epidemiologia. Hipertensão, epidemiologia. Transportes.

Prevalência. Exposição ocupacional. Estudos transversais. Zonas urbanas.

\section{Keywords}

Hearing loss. Noise-induced, epidemiology. Hipertension, epidemiology. Transportation. Prevalence. Occupational exposure. Cross-sectional studies. Urban zones.

\section{Resumo}

\section{Objetivo}

Estimar as prevalências de perda auditiva induzida por ruído e hipertensão arterial em condutores de ônibus urbanos.

\section{Métodos}

Executou-se estudo transversal em amostra probabilística de 108 motoristas da cidade de Campinas, SP. Aplicou-se questionário sobre história profissional, jornadas de trabalho e repouso, e realizou-se exame físico e laboratorial incluindo medida da pressão arterial, audiometria tonal limiar, logoaudiometria e dados antropométricos, após a obtenção de consentimento.

\section{Resultados}

A prevalência de perda auditiva induzida por ruído foi de $32,7 \%$ do total examinado. Segundo a classificação de Merluzzi, nos 31 casos classificados em primeiro e segundo graus, observou-se que a freqüência audiométrica com perda auditiva mais acentuada foi a de $6 \mathrm{kHz}(61,3 \%)$, seguida pela de $4 \mathrm{kHz}(38,7 \%)$, sem diferenças significantes quanto à lateralidade. A prevalência de hipertensão arterial diastólica (PAD $\geq 90 \mathrm{mmHG}$; PAS $\geq 140 \mathrm{mmHG}$ ) foi de $13,2 \%$ dos examinados.

Conclusões

O risco de disacusia induzida por ruído foi maior para os motoristas com mais de seis anos de trabalho, após ajuste para a perda relacionada com a idade, com um odds ratio de 19,25 $(1,59<\mathrm{OR}<386,75 ; \mathrm{p}<0,01)$ para aqueles com mais de 45 anos.

\footnotetext{
Abstract

Objective

To estimate the prevalence of occupational noise-induced hearing loss and arterial hypertension among city bus drivers.

Methods

A cross-sectional study was carried out on a probability random sample of 108 city bus drivers taken out of a total of 1,529 estimated professionals in the city of Campinas, Brazil, in 1991. Drivers were interviewed using questionnaires on job history, shift work and vacation schedules and underwent clinical and laboratory examinations including measures of blood pressure, pure-tone audiometry, speech audiometry, and anthropometric data analysis after an informed consent was obtained.
} 


\section{Results}

The prevalence of noise-induced hearing loss was 32.7\%. According to Merluzzi's classification, $91.2 \%$ (31 cases) were classified as first and second-degree losses and the most affected frequencies were $6 \mathrm{kHz}$ in $61.3 \%$, and $4 \mathrm{kHz}$ in $38.7 \%$. There were no differences in the laterality of the first affected ear. The prevalence of arterial hypertension was $13.2 \%$ (PAD $\geq 90 \mathrm{mmHg} ; \mathrm{PAS} \geq 140 \mathrm{mmHg}$ ).

\section{Conclusions}

The risk of noise-induced hearing loss was greater for drivers in job for more than 6 years, after controlling for age-related hearing loss. An odds ratio of 19.25 was found for those aged 45 or more $(1.59<O R<386.75$; $p<0.01)$.

\section{INTRODUÇÃO}

No ano de 1991, articulou-se projeto cooperativo entre a Universidade Pública, o Programa Público de Assistência à Saúde de Trabalhadores da Prefeitura de Campinas e o sindicato da categoria participante de co-gestão sindical no serviço de saúde. A pesquisa se deu na cidade de Campinas, situada a 100 quilômetros a noroeste da capital do Estado de São Paulo, que contava na época, com uma população de 900 mil habitantes. O modelo assistencial em co-gestão sindical, então vigente para a área de Saúde do Trabalhador no município, atendia a demanda dos trabalhadores com problemas de saúde relacionados com o trabalho, prevalentes em cada categoria profissional. Para isto programou-se um inquérito com amostra populacional aleatória probabilística de motoristas de ônibus urbanos.

As informações disponíveis na literatura em 1991 indicavam a possibilidade de relações epidemiológicas plausíveis entre a prevalência de hipertensão arterial sistêmica (HAS) - definida como um nível de pressão arterial sistólica (PAS) de $140 \mathrm{mmHg}$ ou mais elevada, pressão arterial diastólica (PAD) de $90 \mathrm{mmHg}$ ou mais elevada ${ }^{6}$ - e a prevalência de perda auditiva induzida por ruído (PAIR) - definida segundo o Comitê Nacional de Ruído e Conservação Auditiva* como perda auditiva neurosensorial com lesão nas células do órgão de Corti ${ }^{3}$ - e fatores associados, exposições concomitantes e histórias clínicas individuais de exposição a fatores de risco. Para dar conta da necessidade de informações tão amplas somente um inquérito populacional acompanhado de exames clínicos seria capaz de oferecer dados relevantes.

A prevalência de PAIR em motoristas já foi estudada por Talamini ${ }^{13}$ (1994) em Curitiba. O estudo de Miranda et $\mathrm{al}^{9}$ (1998) também já avaliou a prevalência de PAIR entre trabalhadores setor de transporte da região metropolitana de Salvador, BA.
De acordo com Santos \& Morata $^{12}$ (1999), fatores como o sexo e idade, remédios ototóxicos têm sido descritos como agravantes da perda auditiva induzida pelo ruído. As perdas auditivas também podem estar associadas às doenças na família, otites, meningites além do ruído e pode ser potencializada pelo uso de solventes e metais pesados em ocupações pregressas. ${ }^{5}$

O zumbido é um sintoma que pode ser encontrado no trabalhador portador de PAIR e de acordo ainda com Ferreira Júnior ${ }^{5}$ (1998) o trabalhador pode referir que tem este sintoma nos momentos de repouso auditivo.

Existe consenso na literatura de que o tempo atuando em ocupações de exposição a ruído está associado ao aparecimento da PAIR. No estudo realizado por Cordeiro et $\mathrm{al}^{2}$ (1994) também com motoristas de ônibus em Campinas foi encontrada associação positiva entre a PAIR e o tempo acumulado de trabalho.

Também já foi identificada a associação da PAIR com o tabagismo ${ }^{4}$ c com a hipertensão. ${ }^{14}$

Por precauções éticas e políticas alheias à vontade dos pesquisadores, os resultados do levantamento populacional tiveram sua divulgação retida pelo período de dez anos, que agora se apresenta revisitada pelo exame crítico epidemiológico e pela discussão do impacto social dos achados.

O objetivo do presente estudo foi estudar a prevalência da PAIR e da HAS, em condutores de ônibus urbanos do município de Campinas, SP. A prevalência da PAIR em motoristas caracteristicamente expostos ao ruído é descrita segundo o tempo de profissão, os antecedentes pessoais e a exposição anterior a ruído no ambiente de trabalho e outros ruídos em outros ambientes, segundo a distribuição etária dos motoristas e hábito de fumar. A prevalência de HAS também é descrita segundo o tempo de profissão e a 
exposição anterior a ruído no ambiente de trabalho, distribuição etária e hábito de fumar.

\section{MÉTODOS}

Os trabalhadores em transporte urbano de passageiros do município de Campinas, SP representavam, em 1990, 1.529 motoristas e 1.420 cobradores, os quais são denominados, em conjunto, de condutores de ônibus urbanos.

$\mathrm{Na}$ época do planejamento da pesquisa, existiam em Campinas oito empresas concessionárias de transporte urbano, operando em trajetos baseados em seis terminais de ônibus com início e destino. O número de motoristas variava de 30 a 340 por empresa. Por razões ligadas à qualificação exigida para exercer a ocupação e ao tempo médio de permanência na empresa, optou-se por trabalhar com os motoristas, em um estudo epidemiológico transversal.

A amostra dos motoristas foi selecionada pelo cálculo de quotas proporcionais ao número de motoristas empregados por empresa (Tabela 1). Foi feita uma carta convocatória aos motoristas nos seus locais de trabalho. Aos motoristas que compareceram ao ambulatório foi apresentada uma carta de consentimento para sua participação na pesquisa.

Os motoristas que não compareceram foram reconvocados nominalmente por meio de pedidos aos médicos do trabalho das empresas e ao sindicato da categoria.

Os motoristas das 8 empresas concessionárias trabalhavam em 3 regimes de turnos diferentes, sendo 2 regulares contínuos e 1 descontínuo (e ilegal) com 4 horas durante a madrugada e 4 horas vespertinas, denominado de "picadinho". Isto obrigou à entrega de cartas convite em três horários, nos terminais urbanos - às 8:00, 12:00 e 19:00 horas, respectivamente. Estes horários são os horários de pico, e coincidem com as horas de troca de turnos.

Atribuíram-se pesos 4, 2 e 4 para as possibilidades de um motorista estar trabalhando nos horários de pico para aleatorizar a distribuição de convites. Assim, foi feita a planilha de convocação amostral por empresa e por horário de visita aos terminais.

O cálculo amostral baseou-se em um universo estimado de 1.529 motoristas em atividade nas empresas da cidade. Estimou-se uma freqüência de $30 \%$ para a PAIR (alfa $=5 \%$ e delta $=10 \%$ ) e $10 \%$ para a HAS $(\mathrm{alfa}=5 \%$ e delta $=6 \%)$. Estas proporções foram frutos da observação local (dados não publicados) e da leitura de relatos da bibliografia. Com estes valores concluiu-se que uma amostra de 77 pessoas era adequada para precisar a prevalência da PAIR e 91 pessoas para estimar a hipertensão. Considerando-se perdas em torno de $50 \%$ dos convidados optou-se por selecionar 180 motoristas.

A convocação de motoristas foi feita nos cinco principais terminais de circulação obrigatória de todos os ônibus da cidade. As entrevistas foram agendadas, para aqueles que aceitaram participar, comparecendo ao ambulatório do PST.

Foi estruturada uma seqüência de questionário e exame físico para obtenção de dados dos participantes sobre antecedentes profissionais e da saúde do entrevistado. Aplicou-se o questionário para obter histórico profissional detalhado de exposição ao ruído no ambiente de trabalho, do tempo de profissão dirigindo veículos de transporte de carga e coletivos, do reconhecimento sobre exposições ao ruído em veículos e em outras fontes, inquérito recordatório relativo à ingestão alimentar das últimas 24 horas. Os motoristas participantes também foram submetidos à meatoscopia e audiometria tonal limiar e logoaudiometria, no ambulatório do Programa de Saúde do Trabalhador (PST) da Prefeitura de Campinas.

Foi realizado o exame físico para medir a pressão arterial dos motoristas utilizando manômetro de coluna de mercúrio calibrado para medida zero, tomando três medidas consecutivas, com intervalo mínimo de uma hora, tomadas no braço direito, em posição supina. Como informe associado, mediu-se peso, es-

Tabela 1 - Empresas de ônibus e distribuição proporcional de motoristas na amostra.

\begin{tabular}{lccrrr}
\hline $\begin{array}{l}\text { Empresa } \\
\text { (sigla) }\end{array}$ & $\begin{array}{c}\text { Amostra } \\
\text { N }\end{array}$ & $\begin{array}{c}\text { Total } \\
\%\end{array}$ & $8: 00$ & Amostra em cada horário \\
A & 24 & 13,33 & 9 & 5 & $19: 00$ \\
\hline B & 39 & 21,67 & 16 & 8 & 10 \\
C & 4 & 2,22 & 2 & 5 & 15 \\
D & 28 & 15,56 & 15 & 3 & 1 \\
E & 36 & 20,00 & 6 & 3 & 14 \\
F & 16 & 8,89 & 6 & 4 & 5 \\
G & 14 & 10,78 & 7 & 36 & 8 \\
H & 19 & 100,00 & 72 & & 72 \\
\hline Total & 180 & & & 36 \\
\hline
\end{tabular}


tatura, perímetro do punho e anotou-se a história de jornadas de trabalho no dia anterior com informações qualitativas sobre o sono e o repouso.

Os dados obtidos nos questionários foram digitados em bases eletrônicas que possibilitaram verificar a consistência e corrigir erros por comparação de dupla digitação. Depois de digitados, os questionários foram anexados aos prontuários individuais dos participantes. As análises basearam-se em estatísticas descritivas que geraram pontos de corte para categorizar as variáveis contínuas. As categorias agrupadas foram confrontadas em tabelas de contingência visando a obter estatísticas de homogeneidade a partir do quiquadrado e método exato de Fischer, finalizando-se com análise de associações através de estatísticas de 'odds-ratios'e intervalos de confiança de $95 \%$.

Estudou-se a associação entre os antecedentes ocupacionais e pessoais (exposição ou não) com a PAIR (com perda ou não), descrevendo o número de casos em cada situação e a porcentagem que esse valor representou de pessoas com perda auditiva e sadias entre os expostos e os não-expostos.

\section{Metodologia utilizada para o exame audiométrico}

O exame audiométrico foi realizado em cabine acústica, com audiômetro modelo CAT 741.

Inicialmente foi realizada a meatoscopia. Nos casos de rolha de cerume, a audiometria foi realizada somente após sua remoção (24h após a lavagem otológica).

Foi realizada, em todos, audiometria tonal por via aérea nas freqüências de 250, 500, 1000, 2000, 3000, 4000,6000 e $8000 \mathrm{~Hz}$ em todos os participantes, e por via óssea nas freqüências de 500, 1000, 2000, 3000 e $4000 \mathrm{~Hz}$ naqueles que apresentavam perda auditiva, sempre no período matutino. O repouso acústico foi de 12 horas. Realizou-se ainda a logoaudiometria (limiar de recepção da fala (SRT) e o índice de reconhecimento de fala (IRF). ${ }^{12}$ Os resultados audiométricos foram classificados segundo Merluzzi et $\mathrm{al}^{8}$ (1979).

\section{RESULTADOS}

Dos 108 motoristas amostrados, quatro não realizaram exame audiométrico por condições não apropriadas de repouso prévio e dois não puderam realizar pelo menos duas medidas consecutivas de pressão arterial. Conseqüentemente, as análises de prevalência de HAS foram feitas sobre 106 e a prevalência de PAIR e associação outras variáveis foram conduzidas sobre 104 examinados.

\section{Pressão arterial}

Os resultados foram agrupados em escala de $5 \mathrm{em} 5$ mmHg para os 106 indivíduos com medidas válidas, considerando-se o menor valor de pressões sistólica e diastólica em pelo menos duas aferições consecutivas de pressão arterial.

Considerou-se como hipertenso "lato senso" todo o motorista que apresentasse o menor valor de pressão diastólica igual ou superior a $90 \mathrm{mmHg}$, isto é, aqueles com hipertensão diastólica independentemente da pressão sistólica. Foram observados 14 $(13,2 \%)$ hipertensos (hipertensão diastólica).

\section{Avaliação audiológica}

\section{Audiometria}

Entre os 104 exames audiométricos válidos 62 (59,6\%) apresentavam audição normal e 42 (40,4\%) tinham algum tipo de perda auditiva. Desses últimos, oito apresentavam "Alterações Auditivas não Ocupacionais" e 34 tiveram diagnóstico de "Perda Auditiva Induzida por Ruído", correspondendo a $32,7 \%$ do total examinado.

Os casos de PAIR foram diagnosticados segundo a classificação de Merluzzi, com resultados exibidos na Tabela 2. Foi considerada hipoacusia por ruído de primeiro grau toda perda auditiva neurosensorial na freqüência audiométrica de 4 e/ou $6 \mathrm{kHz}$, com recuperação em $8 \mathrm{kHz}$, uni ou bilateral; e hipoacusia por ruído de segundo grau toda perda auditiva atingindo as freqüências sonoras de 3, 4 e $6 \mathrm{kHz}$, com recupera-

Tabela 2 - Freqüência dos diagnósticos de prevalência de perda auditiva induzida por ruído.

\begin{tabular}{lccr}
\hline Classificação & PAIR & $\begin{array}{c}\text { Freqüência } \\
\text { absoluta }\end{array}$ & $\begin{array}{r}\text { Freqüência } \\
\text { relativa (\%) }\end{array}$ \\
\hline Normal & Não & 62 & 59,6 \\
Hipoacusia p/ ruído de primeiro grau & Sim & 23 & 22,1 \\
Hipoacusia p/ruído de segundo grau & Sim & 8 & 7,7 \\
Déficit p/ ruído + outra causa & Sim & 3 & 2,9 \\
Outras alterações não ocupacionais & Não & 8 & 7,7 \\
\hline Total & & 104 & 100,0 \\
\hline
\end{tabular}

PAIR: Prevalência de perda auditiva induzida por ruído. 
ção em $8 \mathrm{kHz}$. As hipoacusias classificadas como déficit por ruído mais outra causa, também foram consideradas ocupacionais, visto que o trabalho contribuiu como causa da perda.

Nos 31 casos de PAIR, classificados em primeiro e segundo graus, foi realizada uma análise para verificar qual freqüência sonora apresentou perda auditiva mais acentuada. Foram observados 19 casos (61,3\%) em que a essa perda ("entalhe acústico") foi de $6 \mathrm{kHz}$, e 12 casos $(38,7 \%)$ em que a maior perda se deu em $4 \mathrm{kHz}$.

Com referência à lateralidade, das 34 perdas auditivas ocupacionais, observou-se que $15(44,1 \%)$ foram perdas bilaterais simétricas, $1(2,9 \%)$ perda auditiva bilateral assimétrica, mais acentuada na orelha esquerda, $18(52,9 \%)$ perdas unilaterais, sendo $10(29,4 \%)$ na orelha direita e $8(23,5 \%)$ na orelha esquerda, não havendo diferença significativa entre as orelhas.

\section{Logoaudiometria}

Segundo a logoaudiometria, constatou-se não haver comprometimento do índice de reconhecimento de fala em $82 \%$ dos motoristas com PAIR, o que se considerou compatível com os achados audiométricos, ou seja, limiares preservados nas freqüências da fala $(500,1000$ e $2000 \mathrm{~Hz})$

Os resultados obtidos no SRT, em todos os casos, confirmaram os limiares tonais obtidos nas freqüências de 500, 1000 e $2000 \mathrm{~Hz}$.

\section{Critérios para diagnóstico audiológico diferencial}

Considerando-se os antecedentes ocupacionais, os resultados mostraram que $62,5 \%$ dos 104 trabalhadores examinados referiram nunca ter utilizado solventes orgânicos, com ação ototóxica, em qualquer ambiente de trabalho, enquanto que $37,5 \%$ já o haviam utilizado.

Dentre os que usaram algum solvente, o tempo variou de um mês a 10 anos, sendo a média de 2,8 e a mediana de 2,0 anos.

Considerando-se a exposição ocupacional pregressa a metais pesados, $89,4 \%$ dos motoristas nunca a tiveram, enquanto que $10,6 \%$ expuseram-se a metais considerados ototóxicos em funções exercidas anteriormente ao trabalho como motorista. Dos 11 que estiveram expostos a algum tipo de metal pesado, três não referiram o tempo. Entre os oito restantes, o tempo de uso variou de um mês a oito anos.
Quanto aos antecedentes pessoais, 77,9\% dos trabalhadores mencionaram não ter parentes com hipoacusia na família contra $22,12 \%$ que disseram ter.

Dos trabalhadores avaliados, $75 \%$ relataram nunca ter tido otite, enquanto que $22,1 \%$ já haviam tido e três pessoas não fizeram nenhum comentário.

Nenhum dos entrevistados havia sido submetido à cirurgia de orelha anteriormente. Esses dados se confirmaram com a realização da meatoscopia (com a qual se observou ausência de alterações de meato acústico e membrana timpânica), bem como com os achados audiométricos, uma vez que não foram encontradas perdas condutivas.

Quanto ao zumbido, 72,1\% dos indivíduos apresentavam esta característica e $27,9 \%$ não a apresentavam. Esta referência pareceu bastante relevante, uma vez que segundo o Comitê Nacional de Ruído e Conservação Auditiva, o zumbido é um dos sintomas que também caracteriza a PAIR. ${ }^{3}$

Quanto ao uso de arma de fogo, 53,8\% responderam que haviam utilizado, $45,2 \%$ nunca haviam utilizado e um não respondeu.

Os resultados mostraram que houve associação estatística significante entre o uso de metais pesados $(\mathrm{Pb} ; \mathrm{Hg}$; $\mathrm{Cr})$ e a PAIR $(\mathrm{p}=0,036)$, com um odds ratio de 4,28 (IC 95\%:1,01<OR<19,24). Não foi encontrada associação estatisticamente significante entre os demais antecedentes ocupacionais/pessoais e a PAIR; os resultados dos "odds ratios" e seus respectivos intervalos de confiança também não foram significantes.

Dos 19 trabalhadores que tiveram o diagnóstico de PAIR, e que estiveram expostos à arma de fogo, 12 deles apresentaram a "gota acústica" em $6000 \mathrm{~Hz}$.

\section{Tempo de exposição a ruído}

Foi verificado o tempo de exposição a ruídos de 104 indivíduos para os quais foi obtida a avaliação audiológica, agrupando-os, inicialmente, em três classes; posteriormente, houve interesse em combinar essas classes, o que resultou em outras duas, conforme segue:

a) tempo como motorista de ônibus urbano;

b) tempo como motorista de frete ou carga;

c) tempo de exposição a ruído em outras ocupações;

d) tempo como motorista em geral, somando-se os tempos como motorista urbano, de frete e de carga;

e) tempo de exposição a ruído em qualquer ocupação, somando-se os tempos como motorista em 
geral e outras ocupações com exposição a ruído.

Para cada classe foram determinados níveis de exposição, em meses de trabalho, além dos odds ratios para a PAIR e para a HAS (Tabela 3 ).

Os resultados dos testes de associação e seus respectivos valores " $p$ " mostraram que não houve associação estatística significante entre a PAIR e os níveis de exposição nem entre a HAS e os níveis de exposição.

Considerando como exposição a distribuição etária e o hábito de fumar, os resultados dos testes de associação e seus respectivos valores "p" mostraram que houve associação estatística significante entre a PAIR e a faixa etária “acima de 45 anos" $(p=0,0066)$ (Tabela 4).

Foi encontrado risco maior de perda auditiva induzida por ruído para os motoristas com mais de seis anos de trabalho, após ajuste para a perda relacionada com a idade, com um odds ratio de 19,25
$(1,59<\mathrm{OR}<386,75 ; \mathrm{p}<0,01)$ para aqueles com mais de 45 anos.

Foram classificados como "fumantes" todos aqueles que tinham o hábito de fumar na ocasião da entrevista ou que havia deixado de fumar num período de tempo de até cinco anos, nestas condições. Como "ex-fumantes" foram considerados todos os que haviam abandonado o hábito há mais de cinco anos. Os indivíduos classificados como "não-fumantes" foram os que nunca adotaram o tabagismo como hábito. A classificação foi feita com base em informação referida obtida em entrevista.

Para quantificação de tabagismo foi adotada a unidade "anos-maço", que corresponde ao produto do número de maços de 20 cigarros consumidos diariamente pelo tempo (em anos) deste hábito. A média de anos-maço encontrada entre os fumantes foi de 16,4, com mediana de 16,0 .

Quanto à distribuição etária, a idade média dos mo-

Tabela 3 - Odds ratio para a PAIR e para a hipertensão segundo os níveis de exposição ao ruído.

\begin{tabular}{|c|c|c|c|c|c|c|c|}
\hline Variáveis & Categoria & $\underset{N}{\text { Com PAIR }}$ & ${ }_{N}^{\text {Sem PAIR }}$ & $\mathrm{OR}, \mathrm{IC}_{95 \%}$ & $\underset{N}{\text { Com HAS }}$ & $\underset{N}{\text { Sem HAS }}$ & $\mathrm{OR}, \mathrm{IC}_{95 \%}$ \\
\hline \multirow[t]{3}{*}{ TU (meses)* } & Até 24 & 8 & 27 & - & 4 & 31 & - \\
\hline & 24-72 & 11 & 26 & $1,43[0,49 ; 4,13]$ & 3 & 35 & $0,66[0,14 ; 3,20]$ \\
\hline & $>72$ & 15 & 17 & $2,98[1,04 ; 8,52]$ & 7 & 26 & $2,09[0,55 ; 7,93]$ \\
\hline \multirow[t]{3}{*}{$\mathrm{TF}+\mathrm{TC}\left(\right.$ meses) ${ }^{* *}$} & Até 24 & 13 & 20 & - & 3 & 30 & - \\
\hline & 24-72 & 5 & 17 & $0,45[0,13 ; 1,53]$ & 3 & 19 & $1,58[0,29 ; 8,65]$ \\
\hline & $>72$ & 16 & 33 & $0,75[0,30 ; 1,87]$ & 8 & 43 & $1,86[0,46 ; 7,60]$ \\
\hline \multirow[t]{2}{*}{ TEROF (meses)*** } & Até 1 & 11 & 29 & - & 10 & 33 & - \\
\hline & $>1$ & 23 & 41 & $1,48[0,63 ; 3,50]$ & 4 & 59 & $0,22[0,07 ; 0,77]$ \\
\hline \multirow[t]{3}{*}{ TMG (meses) $* * * *$} & Até 120 & 11 & 29 & - & 2 & 38 & - \\
\hline & $121-240$ & 16 & 30 & $1,41[0,56 ; 3,54]$ & 7 & 40 & $3,33[0,65 ; 17,02]$ \\
\hline & $>240$ & 7 & 11 & $1,68[0,52 ; 5,43]$ & 5 & 14 & $6,79[1,18 ; 39,07]$ \\
\hline \multirow[t]{3}{*}{ TERQO (meses) $* * * * *$} & Até 120 & 8 & 19 & - & 2 & 25 & - \\
\hline & $121-240$ & 12 & 34 & $0,84[0,30 ; 2,41]$ & 4 & 43 & $1,16[0,19 ; 6,81]$ \\
\hline & $>240$ & 14 & 17 & $1,96[0,66 ; 5,80]$ & 8 & 24 & $4,17[0,80 ; 21,64]$ \\
\hline \multicolumn{2}{|l|}{ Total } & 34 & 70 & & 14 & 92 & \\
\hline
\end{tabular}

*Tempo de trabalho como motorista de ônibus urbano;

**Tempo de trabalho como motorista de frete ou carga;

***Tempo de exposição a ruído em outras ocupações;

****Tempo como motorista geral, somando-se os tempos como motorista urbano, de frete e de carga

*****Tempo de exposição a ruído em qualquer ocupação, somando-se os tempos como motorista geral e outras ocupações com exposição a ruído;

HAS: pressão arterial sistólica.

Tabela 4 - Odds ratios para a PAIR e para a hipertensão arterial segundo a faixa etária e o hábito de fumar.

\begin{tabular}{|c|c|c|c|c|c|c|c|}
\hline Variáveis & Categorias & ${ }_{N}^{\text {Com PAIR }}$ & ${ }_{N}^{\text {Sem }}{ }_{N}$ & $\mathrm{OR}, \mathrm{IC}_{95 \%}$ & $\underset{N}{\text { Com }}$ HAS & Sem ${ }_{N} \mathrm{HAS}$ & $\mathrm{OR}, \mathrm{IC}_{95 \%}$ \\
\hline \multicolumn{8}{|c|}{ Distribuição etária (anos) } \\
\hline & Até 30 & 4 & 11 & - & 0 & 16 & - \\
\hline & $31-45$ & 17 & 50 & $0,94[0,26 ; 3,33]$ & 8 & 59 & - \\
\hline & $>45$ & 13 & 9 & $3,98[0,95 ; 16,52]$ & 6 & 17 & $2,60[0,68 ; 9,89]$ \\
\hline & Total & 34 & 104 & & 14 & 92 & \\
\hline \multicolumn{8}{|c|}{ Hábito de fumar* } \\
\hline & Não fumante & 6 & 19 & - & 6 & 21 & - \\
\hline & Fumante & 17 & 40 & $2,81[0,75 ; 10,56]$ & 3 & 54 & $0,19[0,04 ; 0,85]$ \\
\hline & Ex-fumante & 8 & 9 & $1,35[0,46 ; 3,96]$ & 5 & 12 & $1,46[0,36 ; 5,81]$ \\
\hline & Total & 31 & 68 & & 14 & 92 & \\
\hline
\end{tabular}

*Cinco pessoas não responderam. 
toristas foi de 38,64 anos e a mediana foi de 37 anos.

\section{DISCUSSÃO}

Para obter-se a autorização patronal para a pesquisa, a postura adotada foi que nenhuma empresa poderia utilizar os dados de uma empresa contra outra ou mesmo conhecer resultados de exames de um empregado seu, sendo divulgados apenas os dados gerais agregados. Neste ponto deixou-se claro para os empresários, que a contrapartida para o segredo que protege os trabalhadores como indivíduos era também resguardar a empresa na sua identidade de mercado. Esta premissa, no entanto, só se mostraria verdadeira se de fato as empresas competissem entre si ao invés de atuar em cartel.

Um aspecto importante foi a discussão com médi$\cos$ do trabalho das empresas, que se revelaram surpresos ao saber que suas fichas e prontuários estavam sendo manipulados por funcionários do serviço de pessoal não obrigados ao segredo.

Ainda durante o período da coleta dos dados, surgiram na cidade clínicas para exame de "Audiometria", com grandes cartazes, indicando que estava começando outro problema: ao invés de prevenir a perda auditiva estava se desencadeando uma epidemia de desemprego e perseguição aos doentes. Houve propostas de interromper-se a pesquisa, mas verificou-se que seria inócuo recuar e ignorar o problema.

Alguns empresários, temendo ações trabalhistas, ameaçavam demitir funcionários com deficiência auditiva, ao invés de desencadear programas de prevenção. Outros "empresários da saúde" vendiam exames de audiometria. Na dúvida, bastava demitir todos e só recontratar aqueles que passassem nos re-exames de empresas particulares de exames médicos. Esta política se prolongou durante o período 1991-2001 com nenhum investimento em programas de conservação auditiva apesar de melhora relativa na situação de conservação e renovação dos modelos dos veículos.

O argumento principal na divulgação dos resultados preliminares foi que o motorista com perda auditiva não é mau motorista. $O$ trânsito da cidade exige controle visual dos carros, das luzes indicadoras e dos passageiros. Um déficit auditivo nas freqüências examinadas não incapacita para dirigir um veículo de carga ou coletivo. Ao contrário, um motorista experiente tem grandes chances $(32,7 \%)$ de ter lesão auditiva provocada pelo trabalho. Este achado aponta para magnitudes semelhantes às encontradas por Talamini ${ }^{13}(1994)$ em Curitiba $(45,2 \%)$ e por Miranda et $\mathrm{al}^{9}$ (1998), em Salvador, BA (29,3\%) que também utilizaram a mesma classificação de Merluzzi em trabalhadores de transportes.

Outro problema foi a reação do sindicato da categoria dos condutores, que tendo lançado como prioridade a luta de denúncia, acabou desencadeando, involuntariamente, maior perseguição contra os próprios colegas dentro das empresas. Por outro lado não existia a certeza do "nexo causal" entre o trabalho na última empresa e a perda auditiva, tendo em vista a complexidade das histórias ocupacionais com exposições múltiplas em várias atividades como motoristas de transporte de carga e de passageiros. A mediana de tempo de trabalho para causar o problema foi de quatro anos e a média foi de 5,4 anos, distribuindo assim a exposição ao risco por vários empregos, impossibilitando responsabilizar diretamente o último, que pode ter sido o menos perigoso.

O inquérito populacional realizado em 1991 visava à produção de conhecimentos dos problemas de saúde que afetavam a categoria dos trabalhadores de ônibus urbanos do Município de Campinas, mediante a análise das prevalências de PAIR e de HAS, de acordo com a demanda sindical apresentada ao Programa de Saúde do Trabalhador.

Os resultados encontrados sugerem que na amostra considerada, a PAIR inicia-se casualmente em uma das orelhas (considerando a hipoacusia de primeiro grau como um estágio inicial da PAIR), passando a ser bilateral com a evolução do quadro, o que se observa no fato de que dos oito indivíduos que apresentaram hipoacusia de segundo grau, sete $(87,5 \%)$ eram bilaterais. Observou-se que o perfil de discriminação vocal dos motoristas é compatível com a comunicação social normal pelo fato de apresentarem perdas auditivas em altas freqüências (primeiro e segundo graus pela classificação adotada), não atingindo, neste estágio, as faixas de freqüência da fala humana.

As prevalências encontradas para a PAIR e para a HAS, foram próximas das encontradas em estudo realizado por Cordeiro et $\mathrm{al}^{2}$ (1994). Em um estudo de prevalência realizado por Miranda et $\mathrm{al}^{9}$ (1998), a partir de dados audiométricos referentes a 7.925 trabalhadores de 44 empresas industriais da região metropolitana de Salvador, a perda auditiva induzida por ruído foi de $35,7 \%$, também muito próxima da encontrada no presente estudo. Em outro estudo de prevalência realizado por Santos et $\mathrm{al}^{11}(1998)$ em uma indústria gráfica a prevalência foi de 39,0\%.

A prevalência de HAS, 13,2\%, obtida no grupo de trabalhadores foi muito próxima daquelas encontradas nos estudos de Cordeiro et al ${ }^{1}$ (1998) e Santana et 
$\mathrm{al}^{10}$ (2001) que trabalharam com outros grupos de profissionais e também àquela estimada para o Brasil (15\%) por Kholmann et $\mathrm{al}^{7}$ (1999).

O presente estudo aponta evidências da influência do tempo de trabalho como motorista urbano na ocorrência da PAIR, o que corrobora com resultado do estudo de Cordeiro et $\mathrm{al}^{2}$ (1994), que também encontrou uma associação entre o tempo de trabalho e a PAIR. No entanto, nossos dados não contribuíram para relacionar o tempo trabalhado como motorista de frete ou carga ou outras ocupações no aumento da prevalência. A falta destas evidências adicionais pode ser tanto devida à baixa intensidade da relação causal em si, como também à insuficiência do tamanho da amostra para evidenciar efeitos menores. Em decorrência, estudos e análises posteriores devem tomar como ponto de partida a maior facilidade para detectar os efeitos do trabalho em ônibus urbanos.

Apesar do tempo de trabalho como motorista acima de 240 meses encontrar-se relacionado à ocorrência da PAIR, este tempo pode estar confundido com a idade dos motoristas, sendo o mesmo válido para a HAS.

Diferentemente do achado de Cruickshanks et $\mathrm{al}^{4}$ (1998) não foi encontrada associação da PAIR com o tabagismo. Também não foram encontradas associações da PAIR com a idade, com doenças pregressas - otite, meningite -, com uso de antibióticos, uso de remédios ototóxicos e uso de solventes apesar dos achados de Santos e Morata ${ }^{12}$ (1999). O zumbido referido em Ferreira Júnior ${ }^{5}$ (1998) não foi associa- do à presença da PAIR, porém o relato do uso de produtos químicos com metais pesados apresentouse associado à PAIR, o que é discutido na literatura sobre o assunto. ${ }^{12}$

Quanto ao risco maior de disacusia (19,25\%) para os motoristas com mais de seis anos de trabalho, fazse necessária uma revisão mais profunda desse resultado uma vez que o Intervalo de 95\% de Confiança para o odds foi muito grande. Os resultados encontrados para as prevalências de PAIR e HAS, bem como qualquer análise conjunta das doenças e seus possíveis fatores de risco, deverão ser cautelosamente estudados levando-se em consideração a redução amostral devida às perdas e também as limitações do desenho de estudo transversal.

O presente trabalho estará sendo retomado em outras comunicações para aprofundar a análise das questões sociais, metodológicas e estatísticas que estão fortemente ligadas aos resultados obtidos.

\section{AGRADECIMENTOS}

À equipe do projeto "PRESSUR", aos vários profissionais que trabalharam no CRST/PST-Campinas por cooperar com a coleta de dados da pesquisa, e, em especial, a Vera Lúcia Salerno; Ana Paula Maranghetti Arias, Ricardo Carlos Cordeiro, Telma Fernandes Barrionuevo Gil Adriana Maria da Silva Orsi Becker, Josely Rimoli, Miriam Pedrolo Silvestre, Sônia Luzia de Souza e Eliane Regina Zampieri de Lima pela colaboração em várias das fases do inquérito.

\section{REFERÊNCIAS}

1. Cordeiro R, Lima Filho EC, Almeida IM. Pressão arterial entre trabalhadores de curtume. Rev Saúde Pública 1998;32:467-76.

2. Cordeiro R, Lima Filho EC, Nascimento LCR. Associação da perda auditiva induzida por ruído com o tempo acumulado de trabalho entre motoristas e cobradores. Cad Saúde Pública 1994;10:210-21.

3. Costa EA, Kitamura S. Órgãos dos sentidos: audição. In: Mendes R. Patologia do trabalho. Rio de Janeiro: Atheneu; 1985. p. 377.

4. Cruickshanks KJ, Klein R, Klein BE, Wiley TL, Nondahl DM, Tweed TS. Cigarette smoking and hearing loss: the epidemiology of hearing loss study. JAMA 1998;279:1715-9.

5. Ferreira Jr-M. Perda auditiva induzida por ruído, PAIR: bom senso e consenso. São Paulo: Editora VK; 1998.

6. Joint National Commitee on Detection, Evaluation, and Treatment of High Blood Pressure. The 1984 report of the Joint National Committee on Detection, Evaluation, and Treatment of High Blood Pressure. Arch Intern Med 1984;144:1045-57.

7. Kholmann Jr-O, Guimarães AC, Carvalho MHC, Chaves Jr-HC, Machado CA, Praxedes JN et al. III Consenso Brasileiro de Hipertensão Arterial. Arq Bras Endocrinol Metab 1999;43:257-86.

8. Merluzzi F, Cornacchia L, Parigi G, Terrana T. Metodologia di esecuzione del controllo dellúdito dei lavoratori esposti a rumore. Nuovo Arch Ital Otol 1979;7:695-714.

9. Miranda CR, Dias CR, Pena PGL, Nobre LCC, Aquino R. Surdez ocupacional em trabalhadores industriais da região metropolitana de Salvador, Bahia. Rev Bras Otorrinolaringol 1998;64:109-14. 
10. Santana VS, Carvalho LC, Santos CP, Andrade C, D'Oca G. Morbidade em candidatos a empregos na região metropolitana de Salvador, Bahia, Brasil. Cad Saúde Pública 2001;17:107-15.

11. Santos LF, Shiraishi NY, Silva JS, Prioste SV, Koishi SM, Lima CJ et al. Estudo da prevalência da perda auditiva induzida por ruído em trabalhadores de uma indústria gráfica. Distúrb Comum 1998;10:45-58.

\section{NOTA DOS AUTORES}

Durante dez anos (1991-2001), não foi possível a divulgação dos resultados da presente pesquisa, por fatores independentes da vontade dos autores. Ainda durante a coleta de dados, chegou ao conhecimento dos pesquisadores que vários condutores tinham deixado o serviço. As empresas conseguiam identificar alguns participantes porque foram fornecidos atestados médicos para justificar a ausência ao trabalho no dia dos exames. A política empresarial vigente não mudou no decorrer da década, tendo sido mantida a alta rotatividade da mão-deobra e os mesmos padrões de exames de saúde para ingresso na função.
12. Santos UP, Morata TC. Efeitos do ruído na audição. In: Santos UP. Ruído: riscos e prevenção. São Paulo: Hucitec; 1999. p. 43-53.

13. Talamini ME. A perda auditiva induzida por ruído em motoristas de ônibus. Tuiuti Ciênc Cul 1994;2:41-56.

14. Talbott E, Helmkamp J, Matthews K, Kuller L, Cotting E, Redmond G. Occupational noise exposure, noiseinduced hearing loss, and the epidemiology of high blood pressure. Am J Epidemiol 1985;121:501-14.

Os atores políticos desta luta foram os próprios motoristas, organizados em sindicatos, as empresas de ônibus, igualmente organizadas em cartel, e os profissionais de saúde das empresas e do município. Houve necessidade de manutenção de sigilo por parte dos pesquisadores. Alguns motoristas foram submetidos a exames audiométricos mais exigentes.

A preocupação imediata do sindicato dos condutores de ônibus e dos pesquisadores foi garantir o segredo sobre a identidade dos sadios e doentes, e o respeito ao direito de abono de faltas eventuais por necessidade de comparecimento aos exames. 